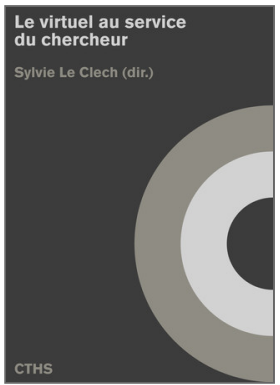

Sylvie Le Clech (dir.)

\title{
Le virtuel au service du chercheur
}

Éditions du Comité des travaux historiques et scientifiques

\section{Introduction}

Nouvelles consciences patrimoniales

\section{Sylvie Le Clech}

DOI : $10.4000 /$ books.cths. 15273

Éditeur : Éditions du Comité des travaux historiques et scientifiques

Lieu d'édition : Éditions du Comité des travaux historiques et scientifiques

Année d'édition : 2021

Date de mise en ligne : 21 juin 2021

Collection : Actes des congrès nationaux des sociétés historiques et scientifiques

EAN électronique : 9782735509300

\section{Sobooks}

http://books.openedition.org

\section{Référence électronique}

LE CLECH, Sylvie. Introduction : Nouvelles consciences patrimoniales In : Le virtuel au service du chercheur [en ligne]. Paris : Éditions du Comité des travaux historiques et scientifiques, 2021 (généré le 24 juin 2021). Disponible sur Internet : <http://books.openedition.org/cths/15273>. ISBN : 9782735509300 DOI : https://doi.org/10.4000/books.cths.15273. 


\title{
Introduction
}

\author{
Nouvelles consciences patrimoniales
}

\author{
Sylvie Le Clech
}

1 Le dernier volume des actes du $144^{\mathrm{e}}$ congrès tenu à Marseille en 2019 traite en dix contributions des relations entretenues entre le réel et le virtuel, dans tous les champs de la mémoire, de l'histoire et des patrimoines. Ces articles associent des commanditaires publics propriétaires d'édifices ouverts à la visite - Chambord, Chinon, Château-Thierry -, des conservateurs, des concepteurs d'outils de représentation et d'aide à la visite, des chercheurs en sciences humaines et sociales.

2 S'ils ont en moins de vingt ans généré de nouveaux usages des publics, des chercheurs professionnels, des citoyens réunis en associations ou s'engageant à titre plus individuel, les rapports entre réel et virtuel ont toujours été l'un des fils conducteurs qui, depuis des périodes plus anciennes, ont permis de produire et d'accéder à la connaissance des faits, des hommes, des objets et des édifices. Cette connaissance intellectuelle, donc virtuelle, s'est arrimée à la matérialité, la réalité des sources d'information, l'existence physique des patrimoines monumentaux et mobiliers - un " déjà là », en quelque sorte. Ce matériau brut de la connaissance, l'objet patrimonial en lui-même, est toujours accompagné de son double virtuel : la dimension symbolique, voire imaginaire, romanesque, immatérielle. La recherche de la signification profonde de cet objet patrimonial dépasse l'exactitude de sa description, de l'esthétique qu'il révèle, de son rattachement à une famille, à un type de patrimoine ou à un espace géographique. Pour les héritiers tardifs que nous sommes, la recherche du sens qu'il a eu pour les hommes du passé reste une "aventure de l'esprit ». C'est ainsi qu'André Malraux qualifiait l'Inventaire général des monuments et richesses artistiques de la France. Le virtuel a donc nativement toute sa place dans cette " aventure de l'esprit », qui n'est que la traduction savante d'une « passion du patrimoine » bien européenne. Depuis les premières fouilles archéologiques de la Renaissance en Italie, la naissance de la discipline historique au $\mathrm{XIX}^{\mathrm{e}}$ siècle, le patrimoine est donc l'objet d'une conscience humaine qui associe le réel - ce qui est mis en évidence - et le virtuel - les hypothèses de recherches qu'aucun chercheur ne saurait entièrement résoudre. 
Cette conscience patrimoniale a pu être douloureuse au XIXe siècle, quand la matérialité des monuments se trouvait en butte aux démolitions; militante $\mathrm{au} \mathrm{xx}^{\mathrm{e}}$ siècle, lorsque cette même matérialité se trouvait menacée par des opérations immobilières dues à l'étalement urbain des Trente Glorieuses; agacée aujourd'hui, quand des politiques publiques semblent se contredire - écologie et culture, transformation énergétique et patrimoine. Cette conscience patrimoniale se prolongeait par des débats dans les œuvres littéraires et scientifiques, qui ont ouvert des possibles et ont donc continué d'entretenir une relation entre réel et virtuel.

5 Notre société du XxI ${ }^{e}$ siècle a profondément bouleversé ces anciennes approches par le recours aux nouvelles technologies, qui peuvent être un des moyens de produire à nouveau des patrimoines immatériels redonnant $d u$ sens et permettant ainsi l'émergence de nouvelles consciences patrimoniales.

6 Ce volume envisage donc le recours aux nouvelles technologies dans des domaines variés : la visite de monuments historiques, la vulgarisation des œuvres conservées dans les grands musées, les expositions virtuelles. Les auteurs cherchent à déterminer si la production de virtuel, utilisé dans des buts de pédagogie et d'accès à la connaissance pour un public élargi, transforme l'expérience de chacun à l'égard de l'existence matérielle d'une œuvre - la magnifiant en quelque sorte, la rendant lisible, en particulier si celle-ci a évolué dans le temps et que sa matérialité ou la connaissance de ses réalités successives comportent interruptions et zones d'ombre. Cette volonté pédagogique est sous-tendue par un discours soit théorique, soit issu de l'expérience empirique des concepteurs de récits, enrichi par les retours des utilisateurs. Notre époque, qui abolit par les usages numériques les phénomènes de distance géographique ou temporelle, utilise le virtuel pour accélérer et massifier un certain nombre de processus de production de données et d'acquisition de connaissances et introduire l'utilisateur dans ce processus. Un nouveau rapport s'établirait-il alors entre les "sachants » et la société civile, par le biais de cette expérience des utilisateurs dans laquelle la recherche de connaissance s'associe au goût du jeu et est aiguisée par des écritures de l'histoire qui se nourrissent de ces usages numériques?

7 Si ce volume envisage le virtuel du point de vue de la médiation comme un point d'aboutissement en aval de la conscience patrimoniale ainsi créée, il appréhende aussi le recours au virtuel bien en amont, du point de vue de la production de nouvelles connaissances et des sciences collaboratives. Y sont étudiés et décryptés, des exemples d'édition numérique de correspondance ou d'indexation de fonds d'archives faisant appel à des professionnels comme à des contributeurs citoyens. On voit alors à l'œuvre de nouvelles méthodes de travail qui enrichissent l'archivistique ou l'édition de textes. Ces deux relations au virtuel, dans leur rapport aux objets patrimoniaux bien réels, associent donc bien finalement les publics, les scientifiques, les concepteurs d'outils de visite et de reconstitution d'une réalité augmentée et les journalistes, qui s'intéressent à l'évolution de la médiation et de la vulgarisation scientifique.

8 Mais l'analyse par l'exemple des rapports entre réel et virtuel ne serait pas complète si le volume n'envisageait pas aussi le cas des patrimoines qui, immatériels par nature gestes, ambiances historiques - ou par destination - la cuisine -, retrouvent forme réelle par une transformation à l'inverse, passant d'un état virtuel à une incarnation réelle. Ainsi en va-t-il des reconstitutions historiques, dans lesquelles l'objet, le vêtement, l'accessoire, bien réels mais chargés d'une dimension symbolique et mémorielle, sont portés par un participant en chair et en os. Le rapport entre le réel et 
le virtuel, dans les cas où ce patrimoine est immatériel car humain, pose alors la question de l'émergence d'une conscience patrimoniale choisie par les personnes ellesmêmes parmi le grand héritage humain. Cette conscience est aussi une «aventure de l'esprit ». Elle donne corps à un imaginaire et traduit une nouvelle identité individuelle ou collective, parfois au prix de petits arrangements avec l'histoire savante. Mais ne dit-on pas que la mémoire est sélective?

\section{AUTEUR}

\section{SYLVIE LE CLECH}

Vice-présidente de la section Histoire du monde moderne, de la Révolution française et des révolutions du CTHS 